\title{
High Sensitive Methane Sensor With Temperature Compensation Based on Selectively Liquid-Infiltrated Photonic Crystal Fibers
}

\author{
Hai LIU*, Haoran WANG, Wen ZHANG, Cancan CHEN, Qing WANG, \\ Yi DING, and Shoufeng TANG
}

School of Information and Control Engineering, China University of Mining and Technology, Xuzhou 221116, China

${ }^{*}$ Corresponding author: Hai LIU_E-mail: lhai_hust@hotmail.com

\begin{abstract}
A highly sensitive and temperature-compensated methane sensor based on a liquid-infiltrated photonic crystal fiber (PCF) is proposed. Two bigger holes near the core area are coated with a methane-sensitive compound film, and specific cladding air holes are infiltrated into the liquid material to form new defective channels. The proposed sensor can achieve accurate measurement of methane concentration through temperature compensation. The sensitivity can reach to $20.07 \mathrm{~nm} / \%$ with a high linearity as the methane concentration is within the range of $0 \%-3.5 \%$ by volume. The proposed methane sensor can not only improve the measurement accuracy, but also reduce the metrical difficulty and simplify the process.
\end{abstract}

Keywords: Photonic crystal fiber; methane sensor; directional resonance coupling; temperature compensation

Citation: Hai LIU, Haoran WANG, Wen ZHANG, Cancan CHEN, Qing WANG, Yi DING, and Shoufeng TANG, "High Sensitive Methane Sensor With Temperature Compensation Based on Selectively Liquid-Infiltrated Photonic Crystal Fibers," Photonic Sensors, 2019, 9(3): 213-222.

\section{Introduction}

Photonic crystal fiber (PCF) [1-3] becomes a great sensing platform due to its special light controlling capability and excellent sensing abilities. With the promotion of fabrication techniques, various PCF-based sensors have been developed for different measurements [4, 5]. One aspect of particular interest in such applications is the target gas detection. Methane is extremely flammable and explosive, and is often found in the gas mixture. Therefore, it is necessary to explore an effective sensing method for the real-time measurement of methane concentration. Many methane-sensing structures have been proposed, including long period fiber grating (LPFG) [6-8], modal interference
(MI) [9], surface plasmon resonance (SPR) [10], cryptophane-E infiltrated photonic crystal (PC) micro-cavity [11], and optical absorption spectroscopy technology [12]. Although these configurations have their own advantages, most of them are interference-sensitive not only to the methane concentration but also to the temperature. The key solution is to eliminate the temperature interference and enhance the measurement precision.

Owing to the distinct advantages of high sensitivity, good physical strength, and low cost, the PCF-based sensor becomes an excellent candidate of methane measurement [13, 14]. However, the temperature cross-sensitivity is still a critical problem to be considered. To solve this problem, the

Received: 29 September 2018 / Revised: 5 January 2019

(C) The Author(s) 2019. This article is published with open access at Springerlink.com DOI: 10.1007/s13320-019-0536-y

Article type: Regular 
combined use of multiple independent waveguides or gratings is commonly utilized $[15,16]$, which would increase the complexity of measurement system. Fortunately, the design flexibility of the PCF facilitates can achieve a multi-channel structure that permits the methane sensing with temperature compensation [17]. Specific cladding air-holes are filled with liquid materials to form defective channels, which introduce new degrees of freedom for multi-parameter sensing. The cross-sensitivity effect can be controlled effectively based on such liquid-infiltrated PCF. Through structural parameter optimization, the methane concentration sensitivity can be up to $20.07 \mathrm{~nm} / \%$ with a good linearity of 0.99999, which is an excellent candidate for gas monitoring.

\section{Theoretical model and parameter optimization}

As shown in Fig. 1, the structural parameters of the proposed PCF are described in detail, including the diameters of air holes $\left(d_{\text {air }}, d_{V_{1}}, d_{V_{2}}, d_{1}\right.$, and $\left.d_{2}\right)$, photonic lattice period $\Lambda$, and the gas-sensitive film thickness $t$. Two smaller holes of the fourth layer with different diameters $d_{V_{1}}$ and $d_{V_{2}}$ are filled with magnetic fluid (MF) materials to introduce different defective channels. The linear relationship between the refractive index (RI) of MF material and temperature can be fitting as $n_{\mathrm{MF}}=1.3418-8.02 \times 10^{-5} \Delta T$ according to related experiment results [18]. Conversely, two bigger holes near the core $\left(d_{b}\right)$ are coated with the methane-sensitive film. Especially, another two different holes of the second layer $\left(d_{1}\right.$ and $\left.d_{2}\right)$ with different diameters are introduced to adjust the coupling intensity of core fundamental mode $\mathrm{LP}_{01}$. The finite element method (FEM) and perfectly matched layers (PML) boundary condition are used to calculate the effective indices of electromagnetic modes [19]. The optimized structural parameters of PCF will be obtained in the end of Section 2.

The whole structure takes into account the practical and convenient qualities to manufacture,

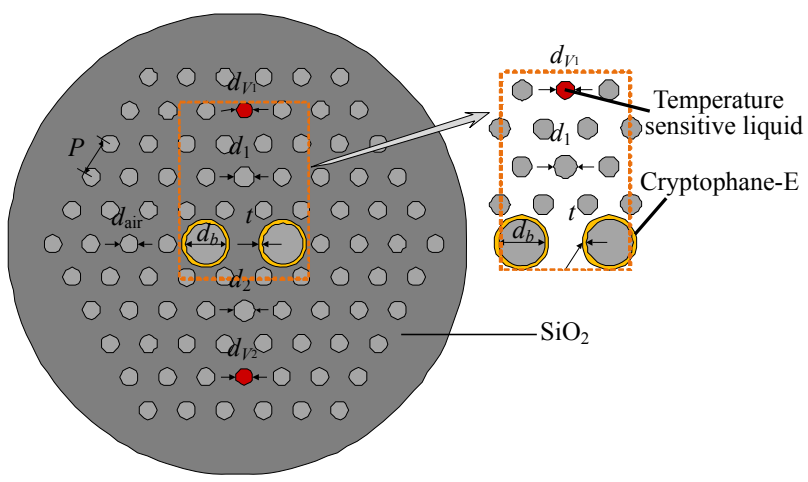

Fig. 1 Cross-section of the PCF.

and the precise fabrication can be ensured through the multi-step "stack-and-draw" method. The collapsing process of silica tubes is carried out in the lathe of modified chemical vapor deposition [20,21]. Capillaries are drawn from high-purity fused silica tubes and stacked layer by layer in a stacking rig, where a capillary is used instead of the solid rod to create a central void in the structure. Moreover, we need to manipulate the pressure in different areas carefully during the whole fiber drawing process. In addition, the precise infiltration of proposed PCF can be achieved through the two-step filling technique [21]. All holes are applied with the ultraviolet (UV)-harden glue through the taped glass tip by carrying a tiny drop of UV-harden glue, except for the ones to be filled. Then, the fiber is connected to a syringe from the other end, and liquid can be pumped into the open holes throughout the whole fiber. Moreover, the uniform thickness methane-sensitive compound film can be coated by a capillary dip-coating technique at a withdrawal speed of $12 \mathrm{~cm} / \mathrm{min}$ [22]. After coating the dilute solution onto the internal surface of air holes, the $0.4 \mathrm{MPa}$ nitrogen stream is used to polish the inner surface of film. Besides, the fusion-splicing machine is used to achieve the splicing between the single mode fiber (SMF) and PCF [23]. During the splicing procedure, the lead-in SMF and PCF should be first aligned roughly center-to-center in the $\mathrm{V}$-grooves [24].

The background material is pure silica, whose material dispersion is determined by the Sellimeiers 
Hai LIU et al:: High Sensitive Methane Sensor With Temperature Compensation Based on Selectively Liquid-Infiltrated Photonic 215 Crystal Fibers

equation as shown in [25]. The RI of methane-sensitive film $n_{\text {methane }}$ varies with the methane concentration $C$ when the methane gas interacts with the intra-cavity compound thin-film $[26,27]$, and the reaction of methane to gas-sensitive film is reversible which is useful for the repeated test. Moreover, based on the experimental results from [28, 29], (2) is selected to describe the relationship between RI of cryptophane-E and gas concentration $C$. Within the range of $0 \%$ to $3.5 \%$, for each $1 \%$ increase in methane concentration, the refractive index of gas-sensitive film will decrease by 0.0046 .

$$
\begin{aligned}
n^{2}= & 1+\frac{0.6961663 \lambda^{2}}{\lambda^{2}-0.0684043^{2}}+ \\
& \frac{0.4079426 \lambda^{2}}{\lambda^{2}-0.1162414^{2}}+\frac{0.8974794 \lambda^{2}}{\lambda^{2}-9.896161^{2}}
\end{aligned}
$$

$$
n_{\text {methane }}=1.448-0.0046 C \text {. }
$$

Figure 2 demonstrates the wavelength dependence of the effective indices of core and defect modes in $x$-polarized direction. Figures 2(a), 2(b), and 2(c) indicate the optical field distribution of core mode $\left(\mathrm{LP}_{01}\right)$ and defect modes $\left(D_{\text {mode } V_{1}}\right.$, $\left.D_{\text {mode } V_{2}}\right) \quad$ at the non-resonant wavelength $\lambda=1480 \mathrm{~nm}, \quad$ respectively. The phase-matching conditions are satisfied when the effective RI of core mode is equal to that of defect modes $D_{\text {mode } V_{1}}$ and $D_{\text {mode_ } V_{2}}$ at the wavelength of $\lambda_{V_{1}}=1393.3 \mathrm{~nm}$ and $\lambda_{V_{2}}=1529 \mathrm{~nm}$. The fiber core and the defective channels can form a balanced directional coupler. At this point, the energy would transfer from the fiber core to defective channel, and a resonant dip would appear in the transmission spectrum. The coupling between the core mode and defect mode ( $D_{\text {mode } V_{1}}$ or $D_{\text {mode } V_{2}}$ ) results from their interaction via their evanescent fields and the coupling process only happens at specific wavelength. The mode coupling between the core mode and defect mode is analyzed by the coupled-mode theory, and the coupling equations are expressed as (3) $[30,31]$. In (3), $\beta_{1}=k_{0} \cdot n_{\text {eff }}^{1}$ and $\beta_{2}=k_{0} \cdot n_{\mathrm{eff}}^{2}$ are the propagation constants of core mode and defect mode, respectively, $k_{0}=2 \pi / \lambda$ is the wave number in free space. Parameters $k_{12}$ and $k_{21}$ are corresponding coupling coefficients, respectively. Besides, $\left|A_{1}\right|^{2}$ and $\left|A_{2}\right|^{2}$ represent the transmitted power of the core mode and defect mode, respectively. Then, the normalized optical powers $P_{1}(Z)$ and $P_{2}(Z)$ can be obtained as (4). In (4), $q=\left(k_{12} k_{21}+\delta^{2}\right)^{1 / 2}$ and $\delta=\left(\beta_{2}-\beta_{1}\right) / 2$, and the initial values are set as $A_{1}(0)=A(0)$ and $A_{2}(0)=0$ as the light is injected into the core only. Since the effective RIs are plural, the complete coupling condition could be satisfied when $\operatorname{Im}\left(n_{\text {eff }}^{1}\right)=\operatorname{Im}\left(n_{\text {eff }}^{2}\right)$.

$$
\begin{gathered}
\frac{d A_{1}}{d z}=\mathrm{i} k_{12} A_{2} \exp \left\{-\mathrm{i}\left(\beta_{2}-\beta_{1}\right) z\right\} \\
\frac{d A_{2}}{d z}=\mathrm{i} k_{21} A_{1} \exp \left\{\mathrm{i}\left(\beta_{2}-\beta_{1}\right) z\right\} \\
P_{1}(Z)=\frac{\left|A_{1}(Z)\right|^{2}}{|A(0)|^{2}}=\frac{k_{12} k_{21}}{q^{2}} \cos ^{2} q z+\frac{\delta^{2}}{q^{2}} \\
P_{2}(Z)=\frac{\left|A_{2}(Z)\right|^{2}}{|A(0)|^{2}}=\frac{\left|k_{21}\right|^{2}}{q^{2}} \sin ^{2} q z .
\end{gathered}
$$

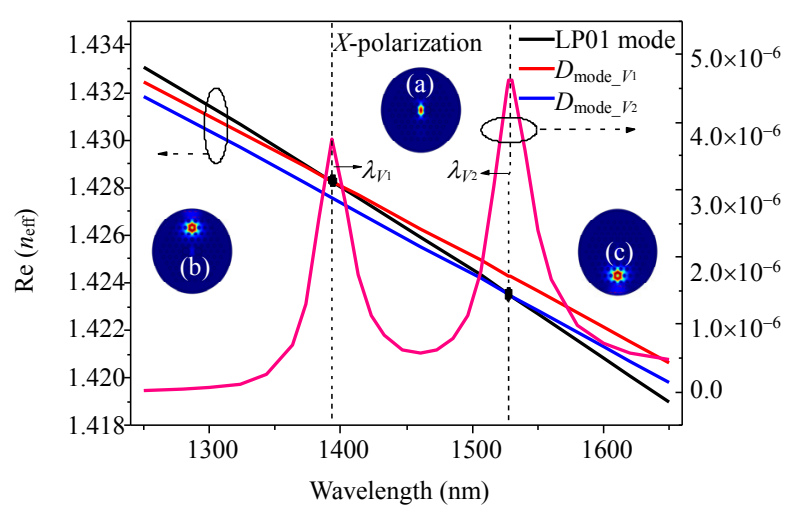

Fig. 2 Curves of effective RIs of $\mathrm{LP}_{01}$ mode, $D_{\text {mode } V_{1}}$, and $D_{\text {mode } V}$ dependent on wavelength and the illustrations representing the optical field distribution of different modes at $\lambda=1480 \mathrm{~nm}$.

Based on the coupled-mode theory, the effective RIs of different modes under the situation of $T=$ $20{ }^{\circ} \mathrm{C}$ are obtained in Fig. 2. Two smaller holes of the fourth layer are with different diameters $d_{V_{1}}$ and $d_{V_{2}}$, so the effective RIs of different defect modes $D_{\text {mode } V_{1}}$ and $D_{\text {mode } V_{2}}$ as function of wavelength are different. It can be seen that the phase-matching conditions are satisfied when the effective RI of core mode is equal to that of defect modes $D_{\text {mode } V_{1}}$ and 
$D_{\text {mode } V_{2}}$ at the wavelength of $\lambda_{V_{1}}=1393.3 \mathrm{~nm}$ and $\lambda_{V_{2}}=1529 \mathrm{~nm}$, respectively. However, at the same time, there is no intersection between $D_{\text {mode } V_{1}}$ and $D_{\text {mode } V_{2}}$. For the $\operatorname{Im}\left(n_{\text {eff }}\right)$ of core mode, there is a sudden change at the point of intersection $\left(\lambda_{V_{1}}\right.$ or $\lambda_{V_{2}}$ ), and two different narrow peaks appear in the curve which can be characterized by confinement loss spectra. In other words, the sensitivity can be measured through analyzing the confinement loss $\left(\alpha_{\mathrm{CL}}\right)$ which can be obtained by (5) as follows [32, 33]:

$$
\alpha_{\mathrm{CL}}(\mathrm{dB} / \mathrm{m})=\frac{20}{\ln 10} \times \frac{2 \pi}{\lambda} \times \operatorname{Im}\left(n_{\mathrm{eff}}\right) .
$$

In order to design a reasonable and available PCF sensor, we need to consider the sensitivity and linearity together. The RI sensitivity of the sensitive film is obtained in Fig. 3, and the sensitivity for $\lambda_{V_{1}}$ and $\lambda_{V_{2}}$ are $4362.32 \mathrm{~nm} / \mathrm{RIU}$ and $4017.6 \mathrm{~nm} / \mathrm{RIU}$, respectively. Figure 4 shows the effect of the structure parameters $t$ and $d_{b}$ on the sensitivity and linearity. The sensitivity of the proposed sensor is changing linearly with the varying of structure parameters $t$ or $d_{b}$, which are summarized in Tables 1 and 2. Then, the gas sensitivities with different $t$ and $d_{b}$ can be obtained as shown in Fig. 5. It is clear that the methane sensitivity will get higher with an increase in the film thickness $t$ and $d_{b}$. For better spectral response, the sensing properties under the condition of $t=0.3 \mu \mathrm{m}$ and $d_{b}=2 \mu \mathrm{m}$ are investigated with the change in these structural parameters, including the photonic lattice period $(P)$, the pitch between adjacent smaller air-holes $\left(d_{\text {air }}\right)$, two larger air-holes $\left(d_{b}\right)$, the diameters of two defective channels $\left(d_{V_{1}}\right.$ and $\left.d_{V_{2}}\right)$ and two specific air-holes $\left(d_{1}\right.$ and $\left.d_{2}\right)$.

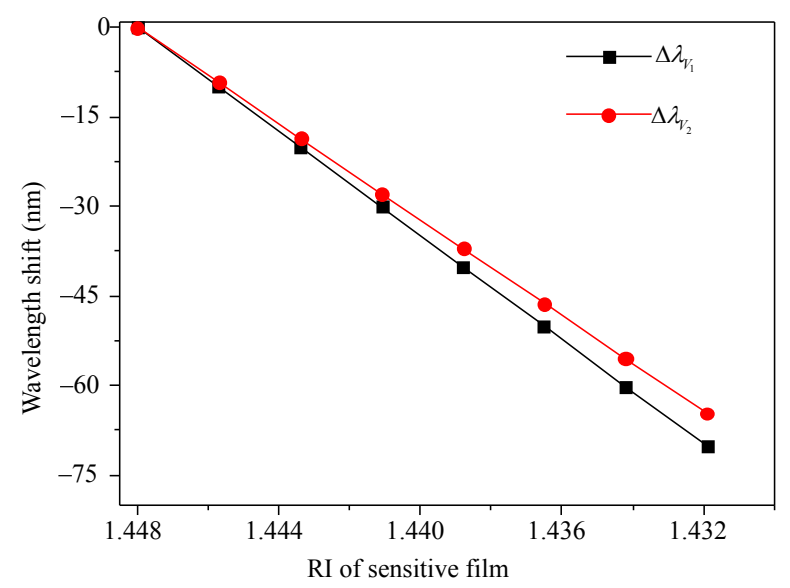

Fig. 3 Effect of sensitive film RI on the sensitivity.

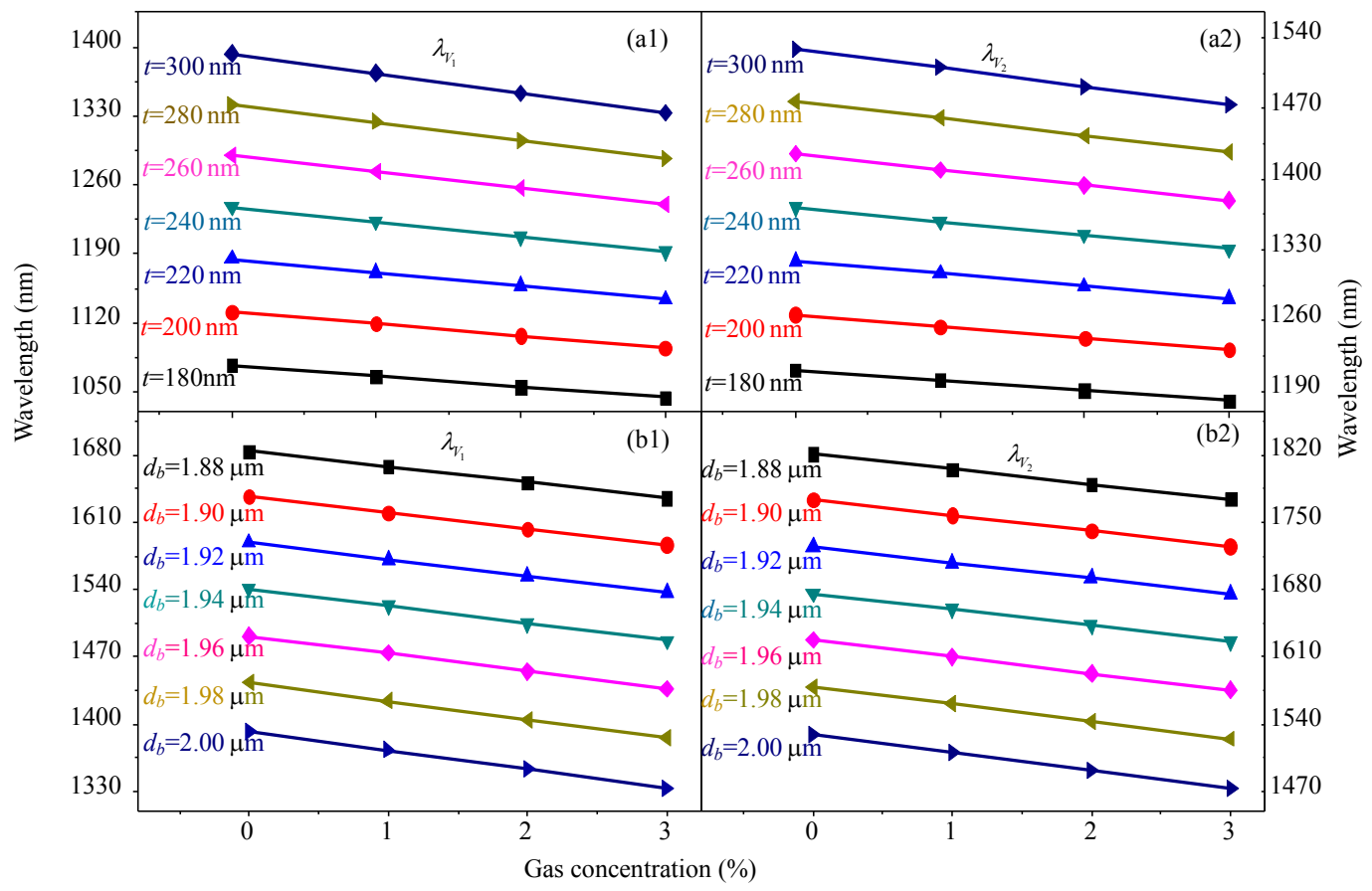

Fig. 4 Methane concentration $C$ related to the resonance wavelength of (a1) $\lambda_{V_{1}}$ at different $t$, (a2) $\lambda_{V_{2}}$ at different $t$, (b1) $\lambda_{V_{1}}$ at different $d_{b}$, and (b2) $\lambda_{V_{2}}$ at different $d_{b}$ under the condition of $T=20^{\circ} \mathrm{C}$. 
Hai LIU et al:: High Sensitive Methane Sensor With Temperature Compensation Based on Selectively Liquid-Infiltrated Photonic 217 Crystal Fibers

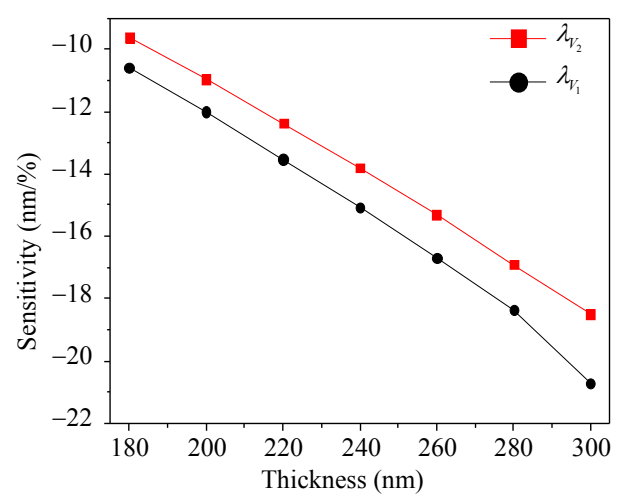

(a)

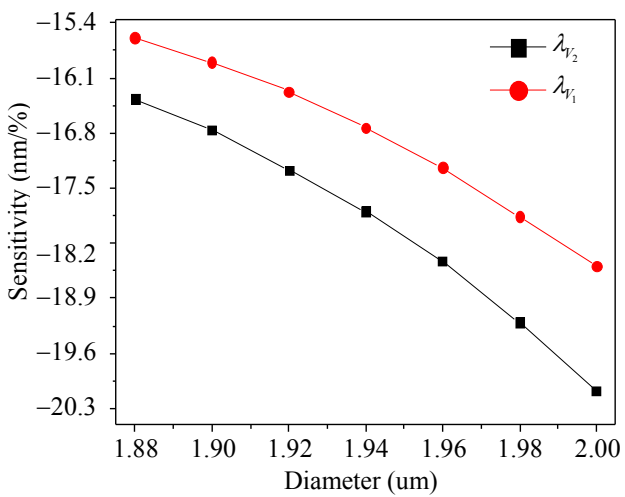

(b)

Fig. 5 Relationship between the gas sensitivity of the sensor and (a) the thickness $t$ of the gas-sensitive film and (b) the diameters $d_{b}$ of air holes.

Table 1 Influence of $t$ on the sensitivity of proposed sensor.

\begin{tabular}{cccc}
\hline$t$-Thickness $(\mathrm{nm})$ & $\lambda_{V_{1}}-$ Sensitivity $(\mathrm{nm} / \%)$ & $\lambda_{V_{1}}-$ Linearity $\left(R^{2}\right)$ & $\lambda_{V_{2}}-$ Sensitivity $(\mathrm{nm} / \%)$ \\
\hline 180 & -10.60 & 0.999 & -9.63 \\
200 & -12.01 & 0.999 & -10.97 \\
220 & -13.54 & 0.999 & -12.39 \\
240 & -15.07 & 0.999 & -13.8 \\
260 & -16.69 & 0.999 & -15.31 \\
280 & -18.36 & 0.999 & -16.90 \\
300 & -20.07 & 0.999 & 0.999 \\
\hline
\end{tabular}

Table 2 Influence of $d_{b}$ on the sensitivity of proposed sensor.

\begin{tabular}{|c|c|c|c|c|}
\hline$d_{b}-$ Diameter $(\mu \mathrm{m})$ & $\lambda_{V_{1}}-$ Sensitivity $(\mathrm{nm} / \%)$ & $\lambda_{V_{1}}-$ Linearity $\left(R^{2}\right)$ & $\lambda_{V_{2}}$-Sensitivity $(\mathrm{nm} / \%)$ & $\lambda_{V_{2}}$ - Linearity $\left(R^{2}\right)$ \\
\hline 1.88 & -16.37 & 0.999 & -15.59 & 0.999 \\
\hline 1.90 & -16.76 & 0.999 & -15.90 & 0.999 \\
\hline 1.92 & -17.27 & 0.999 & -16.27 & 0.999 \\
\hline 1.94 & -17.80 & 0.999 & -16.73 & 0.999 \\
\hline 1.96 & -18.43 & 0.999 & -17.24 & 0.999 \\
\hline 1.98 & -19.20 & 0.999 & -17.86 & 0.999 \\
\hline 2.00 & -20.07 & 0.999 & -18.50 & 0.999 \\
\hline
\end{tabular}

Figure 6 represents the polarization-dependent relationships between the loss-peak shifts and main structural parameters, and the influences of each parameter on the spectral responses are summarized in Table 3. It is interesting to note that the variation of $P$ and $d_{\text {air }}$ can affect the peak-shifts of mode $\lambda_{V_{1}}$ and $\lambda_{V_{2}}$ together, but the parameters $d_{V_{1}}$ or $d_{V_{2}}$ can only affect

the peak-shift separately. Moreover, the variations of $d_{1}$ and $d_{2}$ only affect the peak intensity. Based on above conclusions, we need to find some sort of balance among the central wavelength, loss peak intensity, sensitivity, and linearity. After research and repeated comparisons, the optimized structural parameters of the PCF are presented in Table 4.

Table 3 Influence of different parameters on the loss peak.

\begin{tabular}{|c|c|c|c|c|c|}
\hline \multirow[b]{2}{*}{ Parameter } & \multirow[b]{2}{*}{ Available range $(\mu \mathrm{m})$} & \multicolumn{2}{|c|}{ Peak intensity } & \multicolumn{2}{|c|}{ direction } \\
\hline & & $\lambda_{V_{1}}$ & $\lambda_{V_{2}}$ & $\lambda_{V_{1}}$ & $\lambda_{V_{2}}$ \\
\hline$P$ & $3.18-3.22$ & Increase & Increase & Red-shift & Red-shift \\
\hline$d_{\text {air }}$ & $1.36-1.44$ & Decrease & Decrease & Red-shift & Red-shift \\
\hline$d_{V_{1}}$ & $1.22-1.30$ & Increase & Unchanged & Red-shift & Unchanged \\
\hline$d_{V_{2}}$ & $1.32-1.40$ & Unchanged & Increase & Unchanged & Red-shift \\
\hline$d_{1}$ & $1.56-1.64$ & Decrease & Unchanged & Unchanged & Unchanged \\
\hline$d_{2}$ & $1.66-1.74$ & Unchanged & Decrease & Unchanged & Unchanged \\
\hline
\end{tabular}

Table 4 Values of the optimized parameters.

\begin{tabular}{ccccccccc}
\hline Parameter & $P$ & $d_{\text {air }}$ & $d_{V_{1}}$ & $d_{V_{2}}$ & $d_{1}$ & $d_{2}$ & $d_{b}$ & $t$ \\
\hline Values & $3.2 \mu \mathrm{m}$ & $1.4 \mu \mathrm{m}$ & $1.26 \mu \mathrm{m}$ & $1.36 \mu \mathrm{m}$ & $1.60 \mu \mathrm{m}$ & $1.70 \mu \mathrm{m}$ & $2 \mu \mathrm{m}$ & $0.3 \mu \mathrm{m}$ \\
\hline
\end{tabular}




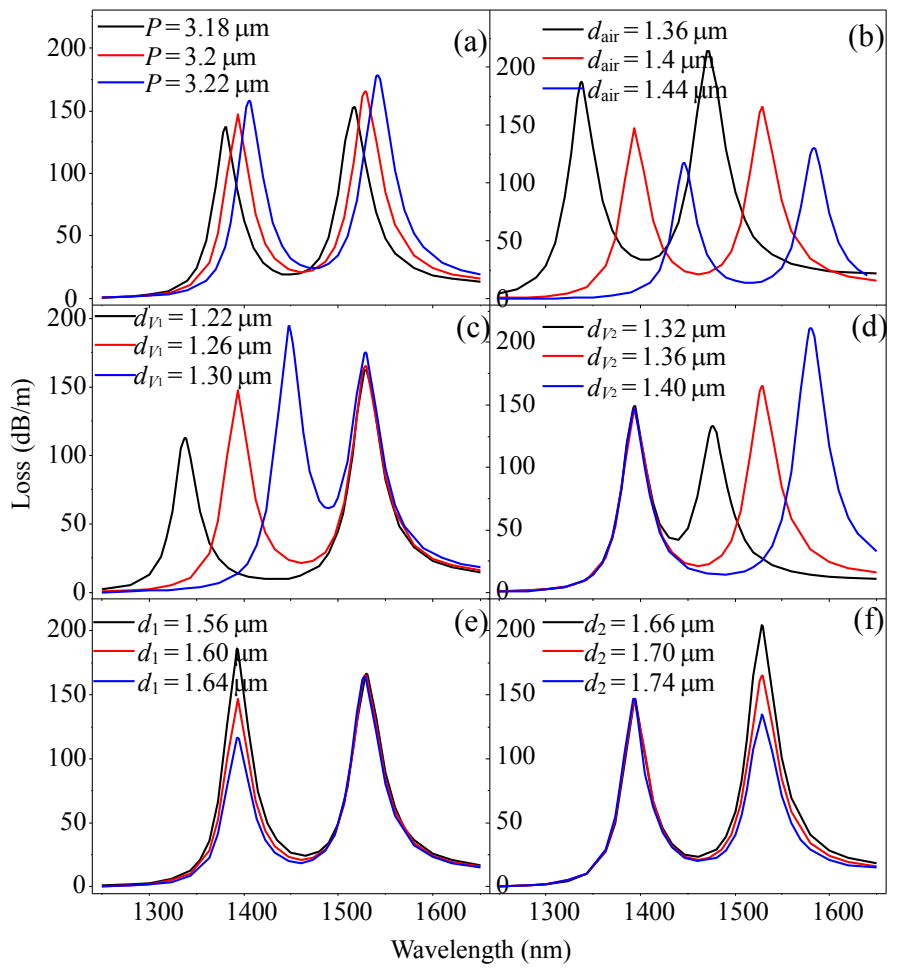

Fig. 6 Loss spectra of the core mode with different values of (a) $P$, (b) $d_{\text {air, }}$, (c) $d_{V_{1}}$, (d) $d_{V_{2}}$, (e) $d_{1}$, and (f) $d_{2}$.

\section{Measurement results and discussion}

The experimental setup begins with an optical broadband source (BBS), and the transmitted light enters the PCF via a polarization controller, as shown in Fig. 7. The spectral response of output light can be observed through an optical spectrum analyzer (OSA). Since the optical properties of the $x$-polarization mode are mainly investigated, a polarization controller (PC) is needed to eliminate the $y$-polarization mode. The concentration of methane gas can be precisely controlled through two mass flow controllers, and the gases are mixed by

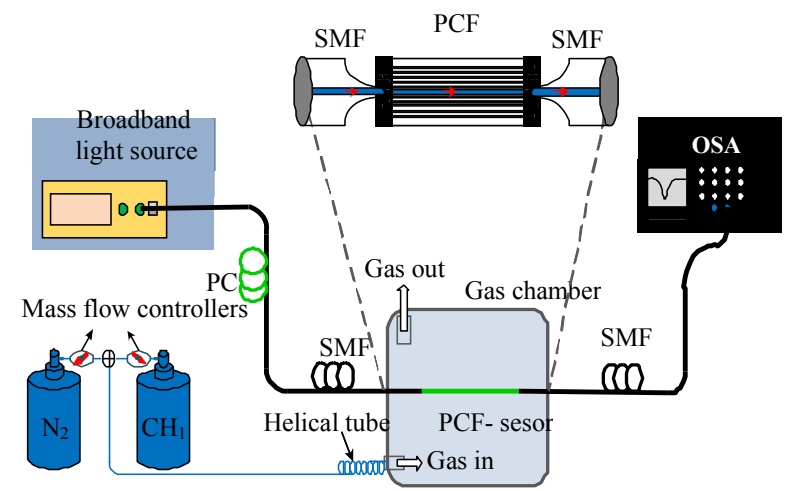

Fig. 7 Principle diagram of the experimental setup. the stainless steel helical tube between the controllers and the chamber. The PCF sensor locates in the center of a controllable gas chamber to evaluate the sensing performance of the methane sensor, and the length of the proposed PCF is set to $18 \mathrm{~mm}$. Moreover, the cladding layer of the SMF at the junctions should be partially removed to make the methane gas enter the air holes easier. The two fiber ends are exposed to an intense discharge for a few seconds, and the fibers should be pushed and pulled to form a robust connection. In order to ensure the accuracy of the measurement, the ventilation time is generally set to 10 minutes before each measurement. Then, the methane gas can enter into the cladding air-holes via free diffusion.

For an accurate simulation, the thermal expansion effect and thermo-optic effect both are considered. The thermal-expansion coefficients are taken as $a_{\text {sio }_{2}}=0.55 \times 10^{-6}$, and the thermo-optic coefficients are chosen as $\xi_{\text {sio }_{2}}=8.3 \times 10^{-6}$. Since the coupling between $\mathrm{LP}_{01}$ mode and $D_{\text {mode }}$ varies with the methane concentration and ambient temperature, the peak-shift of $\mathrm{LP}_{01}$ mode induced by measured 
parameters should be investigated at first. Moreover, based on the experimental results from [34], the sensor's response time is about $50 \mathrm{~s}$, and recovery time is $72 \mathrm{~s}$. Considering the lower explosion limit of methane and the linear relationship between the RI of gas-sensitive film and gas concentration, the methane gas concentration between $0 \%$ and $3.5 \%$ is set as the measurement range. Figure $8(\mathrm{a})$ indicates the confinement loss spectra of $\mathrm{LP}_{01}$ mode within the concentration range of $0 \%$ to $3.5 \%$ on the condition of $T=20{ }^{\circ} \mathrm{C}$, and there is an obvious blue peak-shift with an increase in $C$. The case of $C=0$ is set as the reference point, and then the methane sensitivities for resonant peaks $\lambda_{V_{1}}$ and $\lambda_{V_{2}}$ can be calculated directly. Figure 8(b) reveals the relationship between loss-peak shifts and methane concentration. The gas sensitivities of these two peaks are $k_{1}=-20.07 \mathrm{~nm} / \% \quad$ and $k_{2}=-18.48 \mathrm{~nm} / \%$, respectively. Then, we can obtain the temperature sensitivities in the same way. Figure 9(a) indicates the spectral responses of the device to different temperatures on the condition of $C=0$. Due to a decrease in RI of infiltrated liquid with an increase in temperature $T$, the loss-peaks $\lambda_{V_{1}}$ and $\lambda_{V_{2}}$ have a redshift with an increase in temperature within the range of $0{ }^{\circ} \mathrm{C}$ to $60{ }^{\circ} \mathrm{C}$. Here, the case of $T=0{ }^{\circ} \mathrm{C}$ is selected as the reference point, and the temperature-sensitivity coefficients of $\lambda_{V_{1}}$ and $\lambda_{V_{2}}$ are $k_{3}=0.48 \mathrm{~nm} /{ }^{\circ} \mathrm{C}$ and $k_{4}=0.52 \mathrm{~nm} /{ }^{\circ} \mathrm{C}$, respectively, which can be obtained from Fig. 9(b). The different methane concentration sensitivities and temperature sensitivities are conducive to further multi-parameter discrimination.

According to above results, the loss-peak shifts of $\lambda_{V_{1}}$ and $\lambda_{V_{2}}$ have a good linear relation to the temperature and methane concentration. By the use of matrix demodulation method, the impact of temperature $T$ can be filtered to achieve the accurate measurement of methane concentration $C$. As it is expressed in (6) and (7), the peak-shifts $\Delta \lambda_{1}$ and $\Delta \lambda_{2}$ of $L P_{01}$ depend on the values of $\Delta C$ and $\Delta T$ through the coefficient matrix $\left(k_{1}, k_{2}, k_{3}\right.$, and $\left.k_{4}\right)$.
Based on (8) [32], the sensor resolution of temperature and methane concentration also can be calculated as $4.17 \times 10^{-1}{ }^{\circ} \mathrm{C} .\left(\Delta \lambda_{\min }=0.2 \mathrm{~nm}\right)$ and $9.96 \times 10^{-3} \% \quad\left(\Delta \lambda_{\min }=0.2 \mathrm{~nm}\right)$, respectively.

$$
\begin{gathered}
\left(\begin{array}{l}
\Delta \lambda_{1} \\
\Delta \lambda_{2}
\end{array}\right)=\left(\begin{array}{ll}
k_{1} & k_{3} \\
k_{2} & k_{4}
\end{array}\right)\left(\begin{array}{l}
\Delta C \\
\Delta T
\end{array}\right)=K\left(\begin{array}{l}
\Delta C \\
\Delta T
\end{array}\right) \\
\left(\begin{array}{l}
\Delta C \\
\Delta T
\end{array}\right)=K^{-1}\left(\begin{array}{l}
\Delta \lambda_{1} \\
\Delta \lambda_{2}
\end{array}\right) \\
R=\frac{\Delta T(\Delta C) \cdot \Delta \lambda_{\text {min }}}{\Delta \lambda_{\text {peak }}} .
\end{gathered}
$$

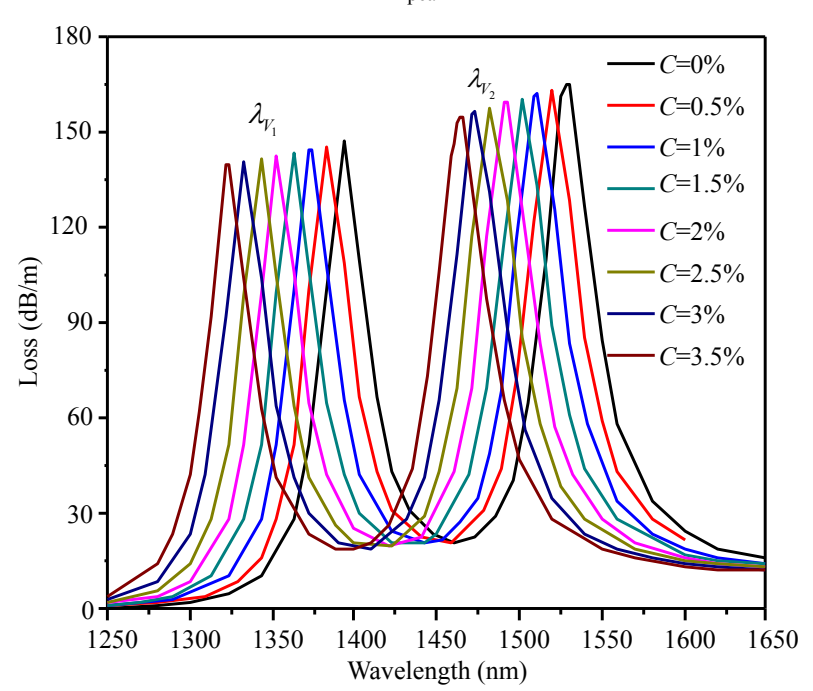

(a)

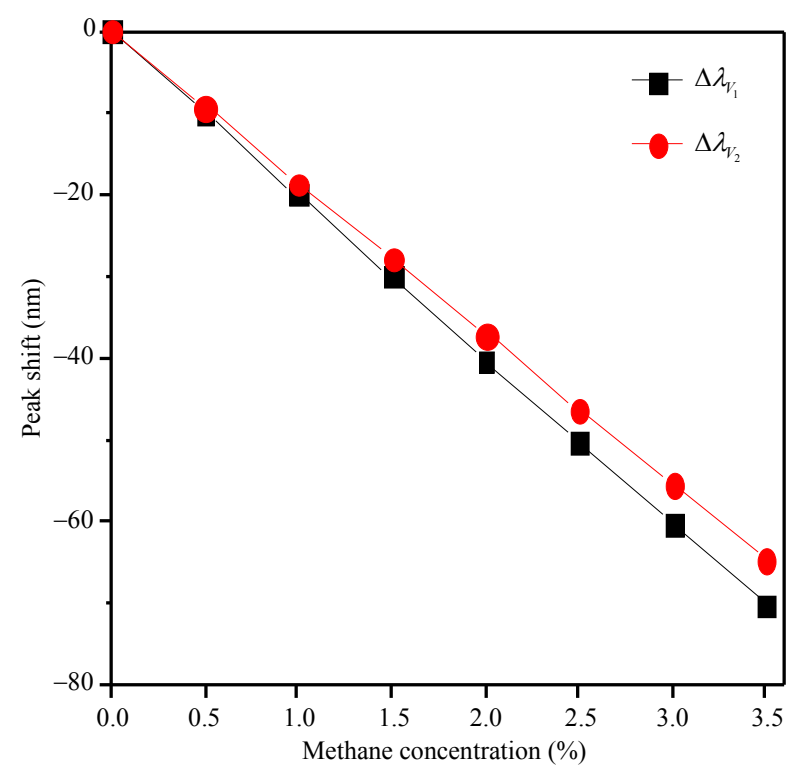

(b)

Fig. 8 Loss spectra and the loss-peak shift with different methane concentration $C$, (a) the loss-peak shift of $\mathrm{LP}_{01}$ mode and (b) the relationship between the loss-peak shift and $C$. 


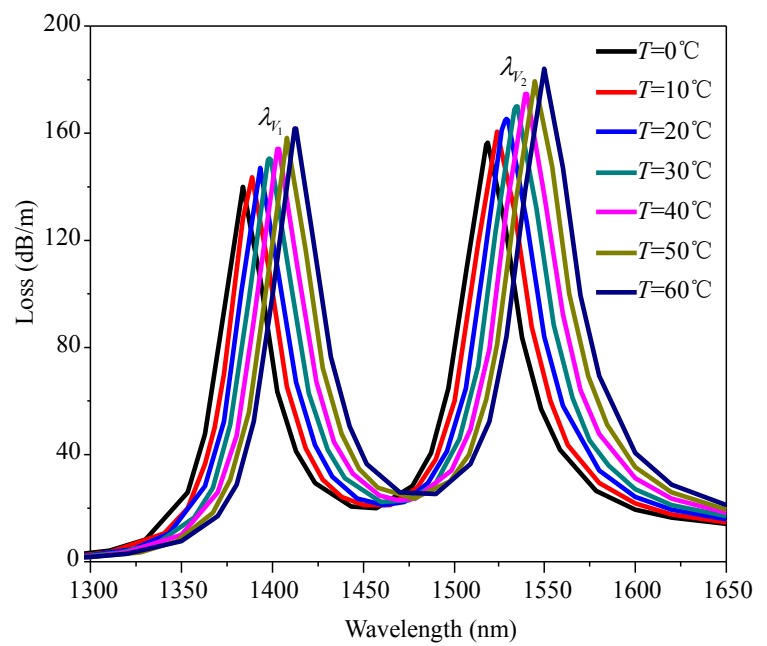

(a)

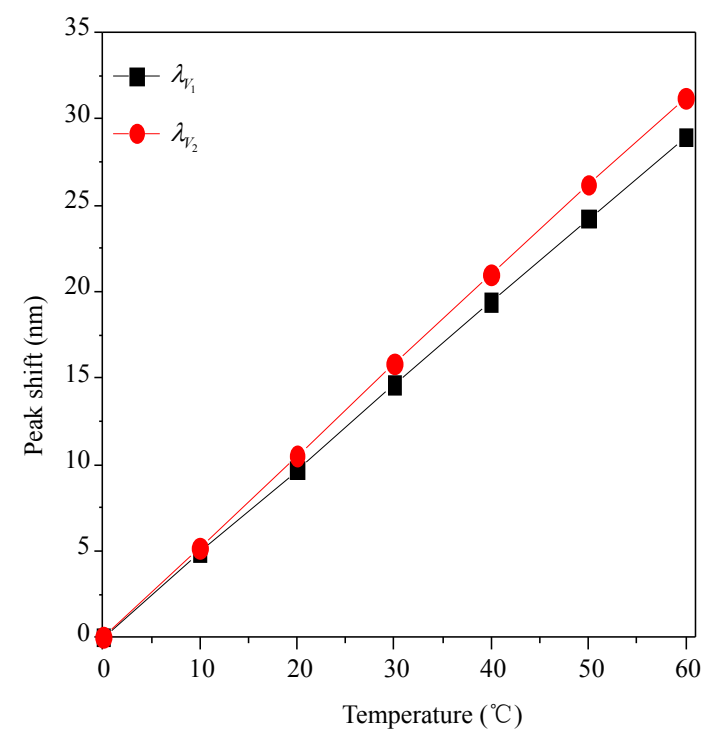

(b)

Fig. 9 Loss spectra and the loss-peak shift with different temperature $T$ : (a) the loss spectral shift of $\mathrm{LP}_{01}$ mode and (b) the relationship between the loss-peak shift and $T$.

A two-dimensional parameter $(C, T)$ is defined to represent the sampling process due to that the ambient conditions vary randomly in the sensing process. Five sampling points $\left[\mathrm{I}\left(1 \%, 30{ }^{\circ} \mathrm{C}\right)\right.$, II $\left(3.3 \%, 7{ }^{\circ} \mathrm{C}\right)$, III $\left(2.1 \%, 50{ }^{\circ} \mathrm{C}\right)$, IV $(2.6 \%$, $\left.45{ }^{\circ} \mathrm{C}\right)$, and $\left.\mathrm{V}\left(1.4 \%, 59{ }^{\circ} \mathrm{C}\right)\right]$ are selected as examples. Based on the loss spectra shown in Fig. 10, the peak-shifts and corresponding results with and without temperature compensation are compared in Table 5. The calculated results show a very good agreement with the theoretical values, and the feasibility and effectiveness of proposed measurement method vary adequately. Especially, the sensor can improve the measurement accuracy by eliminating the temperature cross-sensitivity effect. Table 6 shows the comparison of the sensitivity and detection range of the proposed sensor with some other reported methane sensors. It is obvious that the proposed PCF structure can achieve higher sensitivity.

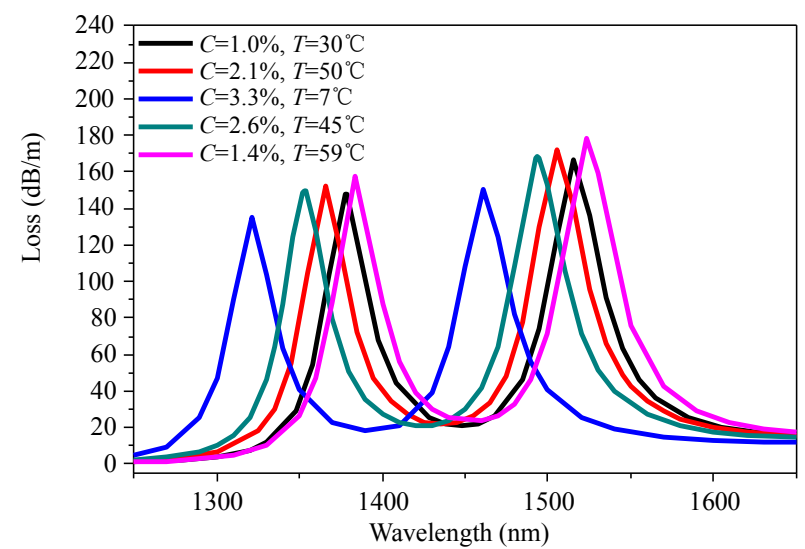

Fig. 10 Loss spectra under five different environmental conditions.

Table 5 Wavelength-shifts with different conditions (States I, II, III, IV, and V) as an example.

\begin{tabular}{ccccc}
\hline $\begin{array}{c}\text { Sampling } \\
\text { points }\end{array}$ & \multicolumn{2}{c}{ Peak shift $(\mathrm{nm})$} & \multicolumn{2}{c}{ Calculated value } \\
\hline$\{C(\%)$, & $\Delta \lambda_{1}$ & $\Delta \lambda_{2}$ & $C$ without & $C$ with \\
$\left.T\left({ }^{\circ} \mathrm{C}\right)\right\}$ & -15.24 & -13.31 & 0.76 & 0.98 \\
$\mathrm{I}\{1,30\}$ & -15 -compensation \\
$\mathrm{II}\{3.3,7\}$ & -72.48 & -67.84 & 3.61 & 3.28 \\
$\mathrm{III}\{2.1,50\}$ & -27.74 & -23.34 & 1.38 & 2.06 \\
$\mathrm{IV}\{2.6,45\}$ & -40.19 & -35.19 & 2.00 & 2.57 \\
$\mathrm{~V}\{1.4,59\}$ & -9.47 & -5.84 & 0.47 & 1.36 \\
\hline
\end{tabular}

Table 6 Comparison of sensing data of various methane sensors.

\begin{tabular}{|c|c|c|c|c|c|}
\hline No. & Type & Sensitivity $(\mathrm{nm} / \%)$ & Low detection limit (ppm) & Correlation coefficient $\left(R_{2}\right)$ & Ref. \\
\hline 1 & PCF-LPG & 0.30 & 2000 & 0.990 & [6] \\
\hline 2 & PCF-LPG & 1.08 & 1800 & 0.998 & [7] \\
\hline 3 & PCF-LPG & 2.5 & 2000 & 0.982 & [8] \\
\hline 4 & Modal interference & 0.51 & 1600 & 0.994 & [9] \\
\hline 5 & (PCF-SPR) & 1.99 & $1005\left(\Delta \lambda_{\min }=0.2 \mathrm{~nm}\right)$ & l & {$[10]$} \\
\hline 6 & PC micro-cavity & 1.67 & 697.35 & / & {$[11]$} \\
\hline 7 & Proposed sensor & 20.07 & $99.65\left(\Delta \lambda_{\min }=0.2 \mathrm{~nm}\right)$ & 0.999 & \\
\hline
\end{tabular}


Hai LIU et al:: High Sensitive Methane Sensor With Temperature Compensation Based on Selectively Liquid-Infiltrated Photonic 221 Crystal Fibers

\section{Conclusions}

In conclusion, a high sensitive methane sensor with temperature compensation based on MF-infiltrated PCF is proposed. The methane concentration can be accurately measured just by using a matrix demodulation method to eliminate the cross-sensitivity effect. The methane sensitivity can be up to $20.07 \mathrm{~nm} / \%$ with a good linearity within the detection range of 0 to $3.5 \%$. The proposed sensor is very suitable for gas measurements owing to its high sensitivity, good linearity, and simple preparation process.

\section{Acknowledgement}

This work was supported by the National Key R\&D Program of China under Grant No. 2016YFC0801800, National Natural Science Foundation of China under Grant No. 51874301, Science and Technology Innovation Project of Xuzhou City under Grant No. KC16SG264, and the Special Foundation for Excellent Young Teachers and Principals Program of Jiangsu Province, China.

Open Access This article is distributed under the terms of the Creative Commons Attribution 4.0 International License (http://creativecommons.org/licenses/by/4.0/), which permits unrestricted use, distribution, and reproduction in any medium, provided you give appropriate credit to the original author(s) and the source, provide a link to the Creative Commons license, and indicate if changes were made.

\section{References}

[1] E. R. Vera, C. M. B. Cordeiro, and P. Torres, "High sensitive temperature sensor using Sagnac loop interferometer based on side-hole photonic crystal fiber filled with metal," Applied Optics, 2017, 56(2): 156-162.

[2] J. S. Wang, L. Pei, S. J. Weng, L. Y. Wu, L. Huang, T. G. Ning, et al., "A tunable polarization beam splitter based on magnetic fluids-filled dual-core photonic crystal fiber," IEEE Photonics Journal, 2017, 9(1): 1-10.

[3] A. A. Rifat, G. A. Mahdiraji, R. Ahmed, D. M. Chow, Y. M. Sua, Y. G. Shee, et al.,
"Copper-graphene-based photonic crystal fiber plasmonic biosensor," IEEE Photonics Journal, 2017, 8(1): 4800408-1-4800408-8.

[4] M. Shi, S. G. Li, and H. L. Chen, “A high-sensitivity temperature sensor based on Sagnac interferometer employing photonic crystal fiber fully filled with ethanol," Applied Physics B, 2018, 124(6): 94-1-94-7.

[5] Q. Liu, S. G. Li, and H. Chen, "Enhanced sensitivity of temperature sensor by a PCF with a defect core based on Sagnac interferometer," Sensors \& Actuators B Chemical, 2018, 254: 636-641.

[6] G. Q. Zhu, X. M. Li, C. Y. Tao, J. Huang, and J. C. Yang, "Optical fiber methane sensor based on SAN film containing cryptophane-E-(OEt)_6," Chinese Optics Letters, 2012, 10(10): 100601-1-100601-3.

[7] J. C. Yang, X. Che, R. Shen, C. Wang, X. M. Li, and W. M. Chen, "High-sensitivity photonic crystal fiber long-period grating methane sensor with cryptophane-A-6Me absorbed on a PAA-CNTs/PAH nanofilm," Optics Express, 2017, 25(17): 20258-20267.

[8] J. C. Yang, L. Zhou, J. Huang, C. Y. Tao, X. M. Li, and W. M. Chen, "Sensitivity enhancing of transition mode long-period fiber grating as methane sensor using high refractive index polycarbonate/cryptophane A overlay deposition," Sensors \& Actuators B: Chemical, 2015, 207: 477-480.

[9] J. C. Yang, L. Zhou, X. Che, J. Huang, X. M. Li, and W. M. Chen, "Photonic crystal fiber methane sensor based on modal interference with an ultraviolet curable fluoro-siloxane nano-film incorporating cryptophane A," Sensors \& Actuators B: Chemical, 2016, 235: 717-722.

[10] H. Liu, M. Wang, Q. Wang, H. W. Li, Y. Ding, and C. H. Zhu, "Simultaneous measurement of hydrogen and methane based on PCF-SPR structure with compound film-coated side-holes," Optical Fiber Technology, 2018, 45: 1-7.

[11] Y. N. Zhang, Y. Zhao, and Q. Wang, "Measurement of methane concentration with cryptophane E infiltrated photonic crystal microcavity," Sensors \& Actuators B: Chemical, 2015, 209(209): 431-437.

[12] Z. F. Wu, C. T. Zheng, Z. W. Liu, D. Yao, W. X. Zhang, Y. D. Wang, et al., "Investigation of a slow-light enhanced near-infrared absorption spectroscopic Gas sensor, based on hollow-core photonic band-gap fiber," Sensors, 2018, 18(7): 2192-1-2192-10

[13] C. Wang, J. C. Yang, R. Shen, X. M. Li, and W. M. Chen, "High-sensitivity photonic crystal fiber long-period grating methane sensor with cryptophane-A-6Me absorbed on a PAA-CNTs/PAH nanofilm," Optics Express, 2017, 25(17): 20258-20267.

[14] M. I. Islam, B. K. Paul, K. Ahmed, M. R. Hasan, S. 
Chowdhury, M. S. Islam, et al., "Highly birefringent single mode spiral shape photonic crystal fiber based sensor for gas sensing applications," Sensing and Bio-Sensing Research, 2017, 14(C): 30-38.

[15] G. F. Yan, L. Zhang, and S. L. He, "Simultaneous measurement of magnetic field and temperature based on an etched TCFMI cascaded with an FBG," Optics Communications, 2016, 364(2): 150-157.

[16] J. X. Wu, Y. P. Miao, B. B. Song, W. Lin, K. L. Zhang, H. Zhang, et al., "Simultaneous measurement of displacement and temperature based on thin-core fiber modal interferometer," Optics Communications, 2015, 340: 136-140.

[17] S. H. Liu, Z. Wang, M. X. Hou, J. Tian, and J. J. Xia, "Asymmetrically infiltrated twin core photonic crystal fiber for dual-parameter sensing," Optics \& Laser Technology, 2016, 82: 53-56.

[18] Y. Zhao, D. Wu, R. Q. Lv, and Y. Ying, "Tunable characteristics and mechanism analysis of the magnetic fluid refractive index with applied magnetic field," IEEE Transactions on Magnetics, 2014, 50(8): 1-5.

[19] K. Saitoh and M. Koshiba, "Full-vectorial imaginary-distance beam propagation method based on a finite element scheme: application to photonic crystal fibers," IEEE Journal of Quantum Electronics, 2002, 38(7): 927-933.

[20] T. Y. Cho, G. H. Kim, K. I. Lee, S. B. Lee, and J. M. Jeong, "Study on the fabrication process of polarization maintaining photonic crystal fibers and their optical properties," Journal of the Optical Society of Korea, 2008, 12 (1): 19-24.

[21] J. Ma, H. H. Yu, X. Jiang, and D. S. Jiang, "High-performance temperature sensing using a selectively filled solid-core photonic crystal fiber with a central air-bore," Optics Experss, 2017, 25(8): 9406-9415.

[22] J. C. Yang, L. Zhou, X. Che, J. Huang, X. M. Li, and W. M. Chen, "Photonic crystal fiber methane sensor based on modal interference with an ultraviolet curable fluoro-siloxane nano-film incorporating cryptophane A," Sensors \& Actuators B: Chemical, 2016, 235: 717-722.

[23] K. Naeem, I. B. Kwon, and Y. Chung., "Multibeam interferometer using a photonic crystal fiber with two asymmetric cores for torsion, strain and temperature sensing," Sensors, 2017, 17(1): 132-1-132-9.

[24] R. Jha, J. Villatoro, and G. Badenes, "Ultrastable in reflection photonic crystal fiber modal interferometer for accurate refractive index sensing,"
Applied Physics Letters, 2008, 93(19): 4057-1-4057-3.

[25] W. Q. Chen, M. D. Thoreson, S. Ishii, A. V. Kildishev, and V. M. Shalaev, "Ultra-thin ultra-smooth and low-loss silver films on a germanium wetting layer," Optics Express, 2010, 18(5): 5124-5134.

[26] M. Benounis, N. J. Renault, J. P. Dutasta, K.Cherif, and A. Abdelghani, "Study of a new evanescent wave optical fibre sensor for methane detection based on cryptophane molecules," Sensors and Actuators B: Chemical, 2005, 107(1): 32-39.

[27] G. Q. Zhu, X. M. Li, C. Y. Tao, J. Huang, and J. C. Yang, "Optical fiber methane sensor based on SAN film containing cryptophane-E-(OEt)6," Chinese Optics Letters, 2012, 10(10): 10-12.

[28] Y. N. Zhang, Y. Zhao, and Q. Wang, "Measurement of methane concentration with cryptophane E infiltrated photonic crystal microcavity," Sensors and Actuators B: Chemical, 2015, 209: 431-437.

[29] X. L. Qian, Y. Zhao, Y. N. Zhang, and Q. Wang, "Theoretical research of gas sensing method based on photonic crystal cavity and fiber loop ring-down technique," Sensors \& Actuators B: Chemical, 2016, 228: 665-672.

[30] D. K. C. Wu, K. J. Lee, V. Pureur, and B. T. Kuhlmey, "Performance of refractive index sensors based on directional couplers in photonic crystal fibers," Journal of Lightwave Technology, 2013, 31(22): 3500-3510.

[31] S. K Varshney, K. Saitoh, R. K Sinha, and M. Koshiba, "Coupling characteristics of multicore photonic crystal fiber-based $1 \times 4$ power splitters," Journal of Lightwave Technology, 2009, 27(12): 2062-2068.

[32] Q. Liu, S. G. Li, H. L. Chen, Z. K. Fan, and J. S. Li, "Photonic crystal fiber temperature sensor based on coupling between liquid-core mode and defect mode," IEEE Photonics Journal, 2015,7(2): 1-9.

[33] Z. H. Zhang, Y. F. Shi, B. M. Bian, and J. Lu, "Dependence of leaky mode coupling on loss in photonic crystal fiber with hybrid cladding," Optics Express, 2008, 16(3): 1915-1922.

[34] B. Shen, P. Q. Yang, X. L. Liu, H. G. Zhang, and S. W. Cao, "Fabrication and characterizations of SAW methane sensor based on cryptophane-E membrane," Journal of Ambient Intelligence and Humanized Computing, 2018: 1-10. 